\title{
Extrahepatic Mucinous Biliary Cystadenoma: A Rare Cause of Gastric Outlet Obstruction
}

\author{
Arkadeep Dhali ${ }^{1}$, Sukanta Ray ${ }^{1}$, Gopal Krishna Dhali ${ }^{1}$, Sujan Khamrui ${ }^{1}$, and Ranajoy \\ Ghosh $^{1}$ \\ ${ }^{1}$ IPGME and R
}

September 24, 2021

\begin{abstract}
Extrahepatic Mucinous Biliary Cystadenoma is an extremely rare clinical entity which can present with varieties of vague clinical signs and symptoms. Gastric outlet obstruction caused due to this has never been reported before. We highlighted the diagnostic dilemma we faced, when radiological investigations couldnot suggest the accurate organ of origin.
\end{abstract}

\section{Introduction:}

Biliary cystadenoma is an extremely rare clinical entity, even more so when located in the extrahepatic biliary tree in comparison to their intrahepatic counterparts [1]. It accounts for less than $5 \%$ of all cystic lesions of liver [2]. Less than 50 cases are reported in existing medical literature. It is typically seen in middle aged female and thought to have hormonal factors like estrogen and progesterone responsible for its pathogenesis [3]. Many a times, it is hard to diagnose due to its vague clinical signs and symptoms, prolonged clinical course and difficult radiological interpretations. Although there are no cases of extrahepatic biliary cystadenomas presenting with gastric outlet obstruction and ours is the first of its kind. The case report was realized according to international Surgical CAse REport (SCARE) guidelines [4].

\section{Case report:}

A 26-year-old female presented to the outpatient department with history of dull-aching, epigastric pain, radiating to the back for last 7 -months. This was accompanied by early satiety and abdominal fullness and progressed to non-bilious vomiting after taking food since last 7-days. There was no history of fever, weightloss, jaundice, or altered bowel habits. There was no relevant past or family history. Physical examination was unremarkable. Laboratory investigations (complete hemogram, serum electrolytes, transaminases, serum bilirubin) were within normal limits. Contrast enhanced computed tomography of the abdomen revealed a fairly enlarged pancreas. A $13.5 \times 10.5 \times 9.3 \mathrm{~cm}$ complex cystic space occupying lesion (SOL) showing septation was seen (Figure 1). Main pancreatic duct was not dilated. Overall it was suggestive of pancreatic cyst. To rule out any intraluminal cause of gastric outlet obstruction, an upper gastrointestinal endoscopy was performed which was unremarkable. An endoscopic ultrasound (EUS) was warranted to better characterise the pancreatic SOL. It showed showed large exophytic multiloculated cystic SOL with mural nodule arising from neck of pancreas (Figure 2). EUS guided aspiration of cyst fluid was done which showed cyst fluid amylase- $23 \mathrm{U} / \mathrm{L}$, CEA- $34.8 \mathrm{ng} / \mathrm{mL}$. Cytology revealed few benign epithelial cells. Considering the provisional diagnosis as cystic neoplasm arising from head of pancreas, Whipple's pancreaticodudenectomy was planned. Intraoperatively, we found distended gallbladder with non-dilated common bile duct along with a 18x10x10 $\mathrm{cm}$ septated cystic mass arising from inferior surface of segments IVB and V of liver (Figure 3). It was having minimal peri-cystic adhesions and was free from hepatoduodenal ligament. After careful adhesiolysis, and dividing the feeding vessels, eneucleation of the mass was done from the liver bed along with cholecystectomy. 
Resected specimen (Figure 4) was sent for histopathological examination. It showed cyst wall lined by cuboidal to columnar epithelium with apical mucin. Dense ovarian type of stroma was seen without any cellular and architectural atypia. Overall it was suggestive of mucinous biliary cystadenoma (Figure 5). She had an uneventful recovery and was discharged on 7th postoperative day. She was found to be well at 18-month follow-up.

\section{Discussion:}

The incidence of biliary cystadenoma ranges between 1:20,000 and 1:100,000, although the malignant counterpart is even rarer with an estimated incidence of 1:10 million [5]. These are generally sporadic in nature [6]. The exact origin of the neoplasm is unknown. Two schools of thought say that either they are derived from ectopic ovarian tissue or from ectopic embryonic gallbladder rests [7]. Estrogen-receptor positivity in the dense ovarian stroma points towards the hormonal dependence in the tumorigenesis [8]. This could very well explain its exclusive occurrence in females. Clinically it can present with non-specific symptoms like epigastric pain, vague abdominal discomfort obstructive jaundice, or even ascending cholangitis [9]. Although these were not the case for us. Here the tumor was big enough to compress the stomach to cause clinical features of gastric outlet obstruction. Moreover as it was extramural and partial obstruction, electrolytes were within normal limits.

Noninvasive imaging modalities like computed tomography (multiloculated lesion, internal septation, papillary projections, intramural nodule, septal enhancement), endoscopic or transabdominal ultrasound helps in characterising the lesion, but a definitive diagnosis can only be attained after histopathological evaluation. It our case these investigations failed to point out the origin of the tumor. Intraoperatively the origin of the tumor was made out and the surgeons took a call to modify the treatment plan as stated above. Preoperative needle aspiration or needle biopsy is not routinely performed as it increases the risk of secondary infection, intra-cystic bleeding, rupture of cyst, needle seeding of tumor cells and dissemination which may complicate the diagnosis and further management. Although in our case the preoperative diagnosis was thought to be pancreatic cystic lesion and differentials considered for that were cystic neoplasms and pseudocyst. Hence cystic fluid aspiration was done to distinguish them.

Surgical excision (complete) is the treatment of choice and other treatment modalities like partial resection, marsupialization, fenestration and sclerosis are shown to be associated with recurrence with a rate which can be as high as $10 \%$ to $90 \%$ [10].

Resected specimen on histological examination gives the definitive diagnosis. The cysts are lined by gastric or biliary columnar epithelial cells with apical mucin. Dense ovarian stroma is also another hallmark finding which suggests benign nature of the disease with good prognosis where as its absence suggests poor prognosis [11]. Immunohistochemistry is also beneficial to better characterise the tumor and is done when the histopathology is inconclusive.

\section{Conclusion}

Extrahepatic biliary cystadenoma is an extremely rare slow-growing tumor that arises predominantly in female and has malignant potential. It presents with vague clinical symptoms and clinicians should aware of it as a differential of gastric outlet obstruction. Moreover they might face a diagnostic dilemma when radiological investigations cannot suggest the accurate organ of origin, which can lead to confusion regarding the treatment to be followed. Hence careful intraoperative evaluation is required before commencing with the procedure. Complete surgical resection remains the treatment of choice to prevent both recurrence and malignant transformation.

Conflicts of interest: None declared.

Financial support: Nil.

Declaration of patient consent: Written informed consent was obtained from the patient for publication of this case report and accompanying images. A copy of the written consent is available for review by the 
Editor-in-Chief of this journal on request.

\section{Author contribution:}

Arkadeep Dhali: Conception, design of the study, acquisition of the data, drafting the manuscript, final approval of the version to be submitted.

Sukanta Ray: Conception, design of the study, acquisition of the data, drafting the manuscript, final approval of the version to be submitted.

Sujan Khamrui: Acquisition of the data, final approval of the version to be submitted.

Ranajoy Ghosh: Acquisition of the data, final approval of the version to be submitted.

Gopal Krishna Dhali: Conception, design of the study, acquisition of the data, drafting the manuscript, final approval of the version to be submitted.

Ethical committee Approval: Not required in our institution to publish anonymous case reports

Provenance and peer review: Not commissioned, externally peer-reviewed.

\section{Reference}

Davies W, Chow M, Nagorney D. Extrahepatic biliary cystadenomas and cystadenocarcinoma. Report of seven cases and review of the literature. Ann Surg . 1995;222(5):619-625. doi:10.1097/00000658-19951100000003

Wang YJ, Lee SD, Lai KH, Wang SS, Lo KJ: Primary biliary cystic tumors of the liver. Am J Gastroenterol 1993, 88:599-603.

Pedram-Canihac M, Le Bail B, Rivel J, Blanc J, Saric J, Bioulac-Sage P: Hepatobiliary cystadenoma with mesenchymal stroma: a hormone dependent tumor. Report of five cases with immunohistochemical study of hormone receptors [Article in French]. Ann Pathol. 2000, 1:14-18.

R.A. Agha, T. Franchi, C. Sohrabi, G. Mathew, The SCARE 2020 guideline: updating consensus Surgical CAse REport (SCARE) guidelines, Int. J. Surg. 84 (2020) 226-230.

Manouras A, Markogiannakis H, Lagoudianakis E, Katergiannakis V (2006) Biliary cystadenoma with mesenchymal stroma: report of a case and review of the literature. World J Gastroenterol 12(37): 60626069.https://doi.org/10.3748/wjg.v12.i37.6062 .

Dixon E, Sutherland FR,Mitchell P,McKinnon G,NayakV (2001) Cystadenomas of the liver: a spectrum of disease. Can J Surg 44(5): 371-376

Lee CW, Tsai HI, Lin YS et al (2015) Intrahepatic biliary mucinous cystic neoplasms: clinicoradiological characteristics and surgical results. BMC Gastroenterol 15:67.https://doi.org/10.1186/s12876-015-0293-3 .

Soares KC, Arnaoutakis DJ, Kamel I, Anders R, Adams RB, Bauer TW, Pawlik TM (2014 Jan) Cystic neoplasms of the liver: biliary cystadenoma and cystadenocarcinoma. J Am Coll Surg 218(1): 119128.https://doi.org/10.1016/j.jamcollsurg.2013.08.014 .

Srinivas T, Bhat SP, Sunder G, Sivarajan R. Mucinous Cystic Neoplasm of the Liver and Extrahepatic Biliary Tract with Ascending Cholangitis: a Case Report and Review of the Literature. Indian J Surg Oncol . 2020;11(Suppl 2):204-207. doi:10.1007/s13193-020-01121-7

Simo KA, Mckillop IH, Ahrens WA, Martinie JB, Ianniti DA, Sindram D (2012) Invasive biliary mucinous cystic neoplasm: a review. HPB: Off J Int Hepato Pancreatol Biliary Assoc 14(11): 725-740.

Soochan D, Keough V, Wanless I, Molinari M (2012) Intra and extra-hepatic cystadenoma of the biliary duct: review of the literature and radiologic and pathological characteristics of a very rare case. BMJ Case Rep 2012:bcr0120125497 

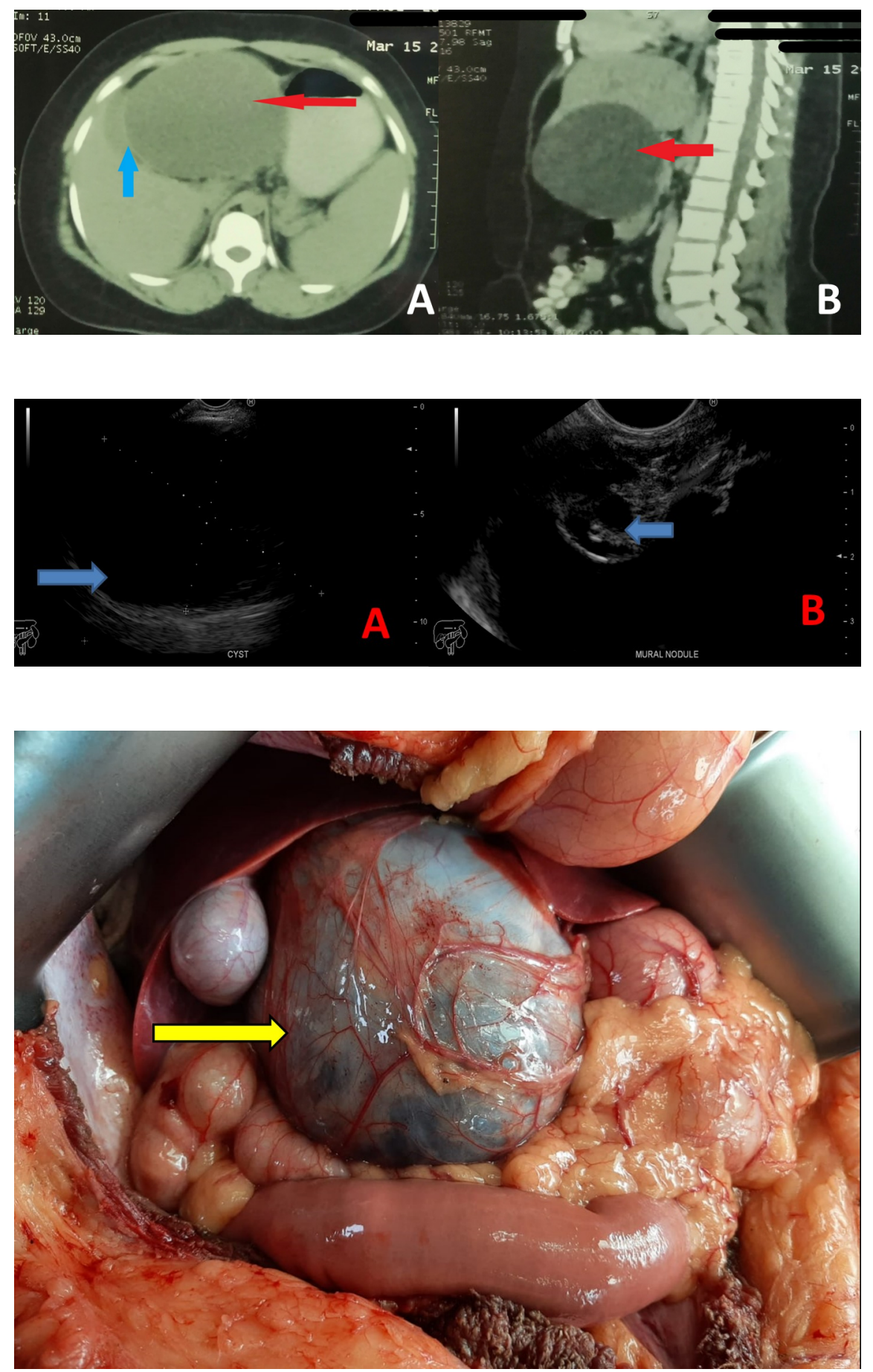

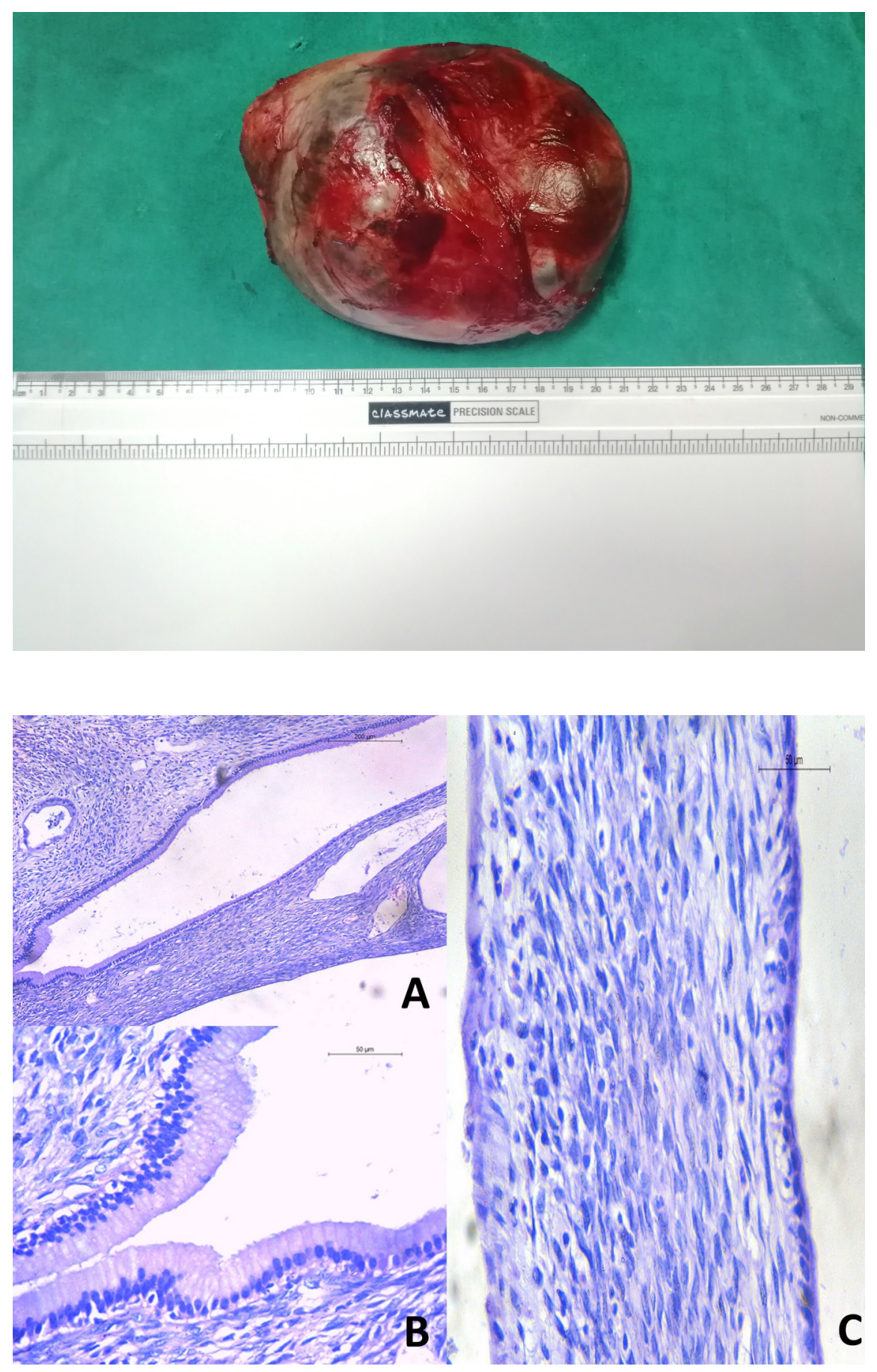